\title{
CLOSED SUBALGEBRAS OF GROUP ALGEBRAS
}

\author{
BY \\ STEPHEN FRIEDBERG $\left(^{(1)}\right.$
}

Introduction. Let $G$ be a compact abelian group with character group $\Gamma$. For basic definitions the reader is advised to see Chapters 1 and 9 of Rudin [7]. Each closed subalgebra $A$ of the group algebra $L^{1}(G)$ induces an equivalence relation on $\Gamma: \alpha \sim \beta$ if and only if $\hat{f}(\alpha)=\hat{f}(\beta)$ every $f$ in $A$, where $\hat{f}$ is the Fourier transform of $f$. We will denote the equivalence classes of $\sim$ by $\left\{E_{\lambda}\right\}$ where $\lambda$ is in some index set and distinguish one special class $E_{0}=\{\gamma \in \Gamma: \hat{f}(\gamma)=0$ for every $f$ in $A\}$ called the zero set of $A$. Since $\Gamma$ is discrete and $\hat{f} \in C_{0}(\Gamma)$ for every $f$ in $L^{1}(G)$, each $E_{\lambda}$, $\lambda \neq 0$, is finite. $E_{0}$ may be infinite.

For $\lambda \neq 0$ let $P_{\lambda}$ be the trigonometric polynomial whose transform is the characteristic function of $E_{\lambda}$, and $A_{0}$ the smallest closed algebra containing every $P_{\lambda}$. Rudin [7, p. 231] has shown that $A_{0}$ induces $\sim$ and is contained in every closed subalgebra which induces $\sim$ with zero set $E_{0}$. If we define $A^{0}$ to be the algebra of all functions whose transforms are constant on every $E_{\lambda}$ and zero on $E_{0}$ then $A^{0}$ will be the largest closed algebra inducing $\sim$.

Rudin [7] asked if there exist distinct closed subalgebras which induce the same equivalence relation. Or, equivalently, does there exist a closed subalgebra $A$ with $A^{0} \neq A_{0}$ ? Kahane [4] gives a negative answer for $G=T$, the circle group.

In $\S 1$ we give sufficient conditions for an equivalence relation on the integers $Z$ to be uniquely induced by exactly one closed subalgebra of $L^{1}(T)$. This result strengthens Theorem 3 of Kahane [4]. We also prove his result on $Z \times Z$.

In $\S 2$ we study the algebras $A_{0}$ and $A^{0}$ in detail and give necessary and sufficient conditions for $A_{0}=A^{0}$.

Finally in $\S 3$ we will consider factorization in closed subalgebras of $L^{1}(G)$ and show that although $L^{1}(G)$ has factorization, there exists a closed subalgebra $A$ without factorization and such that $A \cap L^{p}(G)$ is $L^{1}$-dense in $A$, where $1<p<2$.

0. Notation. If $B$ is a linear subspace of $L^{1}(G)$ we define its annihilator $B^{\perp}=\left\{\phi \in L^{\infty}(G): \phi(f)=0\right.$ for every $f$ in $\left.B\right\}$ where $\phi(f)=\int_{G} f(-x) \phi(x) d x .(d x$ denotes normalized Haar measure.)

Presented to the Society, January 24, 1969; received by the editors January 13, 1969 and, in revised form, April 24, 1969.

( ${ }^{1}$ ) Most of this research is based on a doctoral dissertation that was submitted to Northwestern University under the guidance of Professor Arthur B. Simon. The remainder was done at the Massachusetts Institute of Technology with financial support from the Air Force Office of Scientific Research, Office of Aerospace Research, under Contract No. F44620-67-C-0029. 
Since $G$ is compact we have $L^{\infty}(G) \subset L^{1}(G)$, and it makes sense to consider the Fourier transform of $\phi \in L^{\infty}(G)$, defined $\hat{\phi}(\gamma)=\int_{G} \phi(x) \gamma(-x) d x$ where $\gamma \in \Gamma$. Hence, for a trigonometric polynomial $f=\sum_{i=1}^{n} a_{i} \gamma_{i}, \gamma_{i} \in \Gamma$ we have

$$
\begin{aligned}
\phi(f) & =\int_{G}\left(\sum_{i=1}^{n} a_{i} \gamma_{i}(-x)\right) \phi(x) d x \\
& =\sum_{i=1}^{n} a_{i} \int_{G} \gamma_{i}(-x) \phi(x) d x=\sum_{i=1}^{n} a_{i} \hat{\phi}\left(\gamma_{i}\right) .
\end{aligned}
$$

For $f$ and $g$ in $L^{1}(G)$ we define convolution $(f * g)(x)=\int_{G} f(x-y) g(y) d y$. Thus, with the above notation, it follows that $\phi(f)=(\phi * f)(0)$.

I. Synthesis. In this section we will be concerned specifically with the circle group $T$ and the torus $T^{2}$. $Z$ will denote the group of integers. We will exhibit a class of partitions of $Z$ and $Z \times Z$ which are induced by exactly one closed subalgebra of $L^{1}(T)$ and $L^{1}\left(T^{2}\right)$, respectively.

First, we will generalize Theorem 2 of Kahane [4] on $Z$ and second, we will prove an analagous result on $Z \times Z$.

For the following definition $G$ will be an arbitrary compact abelian group and $\Gamma$ its dual group.

1.1. Definition. An equivalence relation $\sim$ on $\Gamma$ with a distinguished class $E_{0}$ is said to have synthesis if it is induced by exactly one closed subalgebra of $L^{1}(G)$ with zero set $E_{0}$.

Kahane [4] has shown that if $\sim$ is an equivalence relation on $Z$ which satisfies: $n \sim m$ implies $|n-m| \leqq M$, where $M$ is a fixed constant, then $\sim$ has synthesis.

The following is a generalization of Kahane's result.

1.2. TheOREM. Let $\sim$ be an equivalence relation on $Z$ with a distinguished class $E_{0}$. Suppose that for $n, m \notin E_{0}, n \sim m$ and $|n| \leqq|m|$ we have that $|m-n| \leqq M|n|^{1 / 2}(M a$ constant). Then $\sim$ has synthesis.

Proof. It is well known that if $h \in L^{1}(T)$ and is continuous at $x_{0} \in T$, then the Cesaro sums $\left\{U_{n}\right\}$ of its Fourier series $\sum_{j=-\infty}^{\infty} \hat{h}(j) e^{i j x}$ converge to $h$ at the point $x_{0}$. By a standard computation we may write

$$
U_{n}(x)=\sum_{j=-\infty}^{\infty} \hat{K}_{n}(j) \hat{h}(j) e^{i j x}
$$

where $R_{n}(j)=\max (0 ; 1-|j| /(n+1))$. Now let $\sim$ satisfy the above hypotheses. Also, let $A^{0}$ and $A_{0}$ be the maximal and minimal subalgebras of $L^{1}(T)$, respectively, which induce $\sim$. We want to show $A^{0}=A_{0}$ or equivalently, $A^{0 \perp}=A_{0}^{\perp}$.

Obviously, $A^{0 \perp} \subset A_{0}^{\perp}$ so we need only prove the reverse containment. For this purpose, choose $f \in A^{0}$ and $\phi \in A_{0}^{\perp}$. We will show $\phi(f)=0$.

Since $\phi \in L^{\infty}(G)$ and $f \in L^{1}(G)$ we have that $\phi * f$ is continuous. In particular, it is continuous at 0 . We shall approximate $(\phi * f)(0)=\phi(f)$ by the Cesaro sums 
$U_{n}(0)$ of (1) where $h=\phi * f$. In fact, we will show $U_{n}(0) \rightarrow 0$ as $n \rightarrow \infty$. Since $\phi \in A_{0}^{\perp}$ it follows that $\phi\left(P_{\lambda}\right)=0$ or

$$
\sum_{j \in E_{\lambda}} \hat{\phi}(j)=0 .
$$

Define $j_{\lambda}=\min \left\{|j| ; j \in E_{\lambda}\right\}$. Now using (2) and the fact that $\hat{f}$ is constant on every $E_{\lambda}$, we have

$$
U_{n}(0)=\sum_{\lambda \neq 0} \sum_{j \in E_{\lambda}}\left\{\hat{K}_{n}(j)-\hat{K}_{n}\left(j_{\lambda}\right)\right\} \hat{f}(j) \hat{\phi}(j)
$$

where the inner sum extends over those $\lambda$ for which $j_{\lambda} \leqq n$. If $j \in E_{\lambda}$ for such a $\lambda$ we have $\left|j-j_{\lambda}\right| \leqq M j_{\lambda}^{1 / 2}$ or $|j| \leqq j_{\lambda}+M j_{\lambda}^{1 / 2} \leqq(M+1) j_{\lambda} \leqq(M+1) n$. Hence, it follows that $\hat{K}_{n}\left(j_{\lambda}\right)-\hat{K}_{n}(j) \leqq(M+1) / n^{1 / 2}$. Thus, setting $N=(M+1) n$ and applying the Cauchy-Schwarz inequality and the Plancherel theorem to (3) we obtain

$$
\left|U_{n}(0)\right| \leqq \frac{(M+1)}{n^{1 / 2}} \sum_{j=-N}^{N}|\hat{f}(j) \hat{\phi}(j)| \leqq(M+1)\left[\frac{1}{N} \sum_{j=-N}^{N}|\hat{f}(j)|^{2}\right]^{1 / 2}\|\phi\|_{2}
$$

which tends to 0 as $N \rightarrow \infty$ since $\phi \in L^{2}(G)$ and $\hat{f} \in C_{0}(\Gamma)$.

Daniel Rider [6] has an example of an equivalence relation $\sim$ on $Z$ satisfying: $n \sim m,|n| \leqq|m|$ implies $|n-m| \leqq|n|$, and does not have synthesis.

This yields the following interesting question which we have not been able to answer: Suppose $\sim$ is an equivalence relation on $Z$ satisfying: $n \sim m,|n| \leqq|m|$ implies $|n-m| \leqq M|n|^{\delta}$, where $M$ is some constant and $1 / 2<\delta<1$. For which values of $\delta$ does $\sim$ have synthesis?

The more ambitious task of classifying all synthetic partitions is beyond our reach. However, suppose $S$ is a "Sidon set" (see [7, pp. 121-130] for the definition and facts about Sidon sets) and $A$ is a closed subalgebra of $L^{1}(T)$ with $Z-E_{0} \subset S$. Then $A^{0}=A_{0}$. In fact, it will follow that if $B$ is a closed subalgebra of $A$ then $B^{0}=B_{0}$.

The problem of discovering synthetic equivalence relations on $Z \times Z$ is open. Although we will prove an extension of Kahane's result to $Z \times Z$, the correspondingly stronger result of (1.2) has eluded us.

We begin with some facts and definitions which may all be found in Zygmund [8, Vol. II].

The torus will be denoted $T^{2}$. Let $R(n)=\{(i, j) \in Z \times Z:|i| \leqq n,|j| \leqq n\}$. For $h \in L^{1}\left(T^{2}\right)$ let

$$
S_{n}(\bar{x})=\sum_{j \in R(n)} \hat{h}(j) e^{i(j \cdot x)}
$$

where $\bar{x} \in T^{2}$, and - represents the usual dot product.

By a standard manipulation we may write the Cesaro sums $\left\{U_{n}(\bar{x})\right\}$ of (1) by

$$
U_{n}(\bar{x})=\frac{1}{(n+1)^{2}} \sum_{j \in R(n)}\left(n-\left|j_{1}\right|+1\right)\left(n-\left|j_{2}\right|+1\right) \hat{h}(\bar{j}) e^{i(j \cdot x)}
$$

where $j=\left(j_{1}, j_{2}\right)$. 
1.3. Theorem [8, Vol. II, P. 304]. Suppose $h \in L^{\infty}\left(T^{2}\right)$ and $h$ is continuous at $\bar{x}_{0} \in T^{2}$. Then $\left|h\left(\bar{x}_{0}\right)-U_{n}\left(\bar{x}_{0}\right)\right| \rightarrow 0$ as $n \rightarrow \infty$.

1.4. THEOREM. Let $\sim$ be an equivalence relation on $Z \times Z$ which satisfies; $\left(n_{1}, m_{1}\right)$ $\sim\left(n_{2}, m_{2}\right)$ implies $\left|n_{1}-n_{2}\right| \leqq M$ and $\left|m_{1}-m_{2}\right| \leqq M$ for $\left(n_{i}, m_{i}\right) \notin E_{0}, i=1,2$, where $M$ is some constant. Then $\sim$ has synthesis.

Proof. The proof is similar to that of (1.2). We define

$$
\hat{K}_{n}(i, j)=\max (0 ; 1-|i| /(n+1)) \max (0 ; 1-|j| /(n+1))
$$

$i_{\lambda}=\min \left(|i| ;(i, j) \in E_{\lambda}\right)$, and $j_{\lambda}=\min \left(|j| ;(i, j) \in E_{\lambda}\right)$. Now if $\phi \in A_{0}^{1}, f \in A^{0}$ we let $h=\phi * f, \bar{x}_{0}=0$ and rewrite (2) as

$$
\begin{aligned}
U_{n}(0) & =\sum_{(i, j) \in Z \times Z} \hat{K}_{n}(i, j) \hat{\phi}(i, j) \hat{f}(i, j), \\
& =\sum_{\lambda \neq 0} \sum_{(i, j) \in E_{\lambda}}\left\{\hat{K}_{n}(i, j)-\hat{K}_{n}\left(i_{\lambda}, j_{\lambda}\right)\right\} \hat{f}(i, j) \hat{\phi}(i, j)
\end{aligned}
$$

where the inner sum extends over those $\lambda$ for which $i_{\lambda} \leqq n$ and $j_{\lambda} \leqq n$. If $(i, j) \in E_{\lambda}$ for such a $\lambda$ then $|i| \leqq(M+1) n$ and $|j| \leqq(M+1) n$. Hence $\hat{K}_{n}\left(i_{\lambda}, j_{\lambda}\right)-\hat{K}_{n}(i, j) \leqq C / n$ where $C$ is a constant independent of $n$. Thus, as in the proof of (1.2) we have

$$
\left|U_{n}(0)\right| \leqq \frac{C}{n}\left[\sum_{(i, j) \in R(n)}|\hat{f}(i, j)|^{2}\right]^{1 / 2}\|\phi\|_{2}
$$

which tends to 0 as $n \rightarrow \infty$.

II. The algebras $A_{0}$ and $A^{0}$. Throughout this section, $G$ will be an arbitrary compact abelian group and $A$ a closed subalgebra of $L^{1}(G)$ with induced equivalence relation $\sim$ and zero set $E_{0}$. We will be concerned with finding conditions which will guarantee $A_{0}=A^{0}$. It will be shown in (2.1) that although $A_{0}$ and $A^{0}$ have the same maximal ideal space, their annihilators $A_{0}^{\perp}$ and $A^{0 \perp}$ respectively, can be characterized in (2.3) quite simply. We will construct a linear subspace $B$ of $L^{\infty}(G)$ consisting of trigonometric polynomials such that $A^{0 \perp}=w^{*}$-closure of $B$ and $A_{0}^{\perp}=\left(L^{2}\right.$-closure of $\left.B\right) \cap L^{\infty}(G)$.

Denote the maximal ideal space of $A$ by $\Delta(A)$ and let $\Gamma^{\prime}$ be the quotient space obtained from $\Gamma \backslash E_{0}$ by identifying equivalent elements of $\Gamma$. If we let $\gamma^{\prime}$ be the image of $\gamma$ under the quotient map, it is clear that $\gamma^{\prime}$ induces a nonzero homomorphism $h$ on $A$ defined by $h(f)=\hat{f}(\gamma)$ for $f \in A$. However, the converse is also true.

2.1. THEOREM. Every nonzero homomorphism on $A$ is induced by an element of $\Gamma^{\prime}$.

Proof. Define $T: A \rightarrow C_{0}\left(\Gamma^{\prime}\right)$ by $T(f)\left(\gamma^{\prime}\right)=\hat{f}(\gamma)$. It is easy to show that $T\left(A_{0}\right)$ is a separating, conjugate-closed subalgebra of $C_{0}\left(\Gamma^{\prime}\right)$. Since $A_{0} \subset A$ (Rudin [7, p. 232]) we have by the Stone-Weierstrass theorem that $T(A)$ is dense in $C_{0}\left(\Gamma^{\prime}\right)$ under the uniform norm. It follows that the adjoint map $T^{*}: \Gamma^{\prime} \rightarrow \Delta(A)$ carries 
$\Gamma^{\prime}$ homeomorphically onto a closed subset of $\Delta(A)$ (see for example [5, p. 76]). It remains to show $T^{*}\left(\Gamma^{\prime}\right)=\Delta(A)$. Choose $h \in \Delta(A)$. Then for $f \in A$

$$
|h(f)|^{n}=\left|h\left(f^{n}\right)\right| \leqq\left\|f^{n}\right\|_{A}=\left\|f^{n}\right\|_{L^{1}(G)} .
$$

By the spectral radius formula we obtain

$$
|h(f)| \leqq\|\hat{f}\|_{C_{0}(\Gamma)}=\|\hat{f}\|_{C_{0}\left(\Gamma^{\prime}\right)} .
$$

Hence, the function $h^{\prime}(T(f))=h(f)$ is a continuous homomorphism on $T(A)$ and may be extended to all of $C_{0}\left(\Gamma^{\prime}\right)$ since $T(A)$ is dense in $C_{0}\left(\Gamma^{\prime}\right)$. Thus, there exists $\gamma^{\prime} \in \Gamma^{\prime}$ with $h(f)=T^{*}\left(\gamma^{\prime}\right) f$ for every $f \in A$. Consequently, $T^{*}$ is onto.

The above theorem suggests that Banach algebra methods of distinguishing $A_{0}$ and $A^{0}$ will be of little help. Also, the fact that $A_{0}$ is generated as a vector space as well as an algebra by the $P_{\lambda}$ leads us to study the annihilators $A_{0}^{\perp}$ and $A^{0 \perp}$. For this purpose we introduce the following notation.

Let $\sim$ have equivalence classes $\left\{E_{\lambda}\right\}$. For $\lambda \neq 0$ and $E_{\lambda}=\left\{\gamma_{1}, \ldots, \gamma_{n}\right\}$ define

$$
\begin{aligned}
B_{\lambda}=\left\{\phi \in L^{\infty}(G): \phi=\sum_{i=1}^{n} a_{1} \gamma_{i} \text { where } a_{1}, \ldots,\right. & a_{n} \text { are complex } \\
& \text { numbers with } \left.\sum_{i=1}^{n} a_{i}=0\right\}
\end{aligned}
$$

and let $B_{0}$ be the linear space of $L^{\infty}(G)$ generated by the elements of $E_{0}$. (If $E_{0}=\varnothing$, let $B_{0}=\varnothing$.) Finally, let $B$ be the linear space of $L^{\infty}(G)$ generated by the elements of $\bigcup B_{\lambda}$.

2.2. Lemma. For any closed subalgebra $A$ of $L^{1}(G)$, we have $B \subset A^{\perp}$.

Proof. Pick $f \in A$ and an index $\lambda$. If $\lambda \neq 0$ and $\phi \in B_{\lambda}$, then $\phi=\sum_{i=1}^{n} a_{i} \gamma_{i}$ with $\sum_{i=1}^{n} a_{i}=0$ where $E_{\lambda}=\left\{\gamma_{1}, \ldots, \gamma_{n}\right\}$. Then

$\phi(f)=\int f(x) \phi(-x) d x=\int f(x) \sum_{i=1}^{n} a_{i} \gamma_{i}(-x) d x=\sum_{i=1}^{n} a_{i} \hat{f}\left(\gamma_{i}\right)=\hat{f}\left(E_{\lambda}\right) \sum_{i=1}^{n} a_{i}=0$.

If $\lambda=0$ and $\phi \in B_{0}$, then $\phi=\sum_{i=1}^{k} b_{i} \beta_{i}$ with $\beta_{i} \in E_{0}$ for $i=1, \ldots, k$. Then, similarly, $\phi(f)=\sum_{i=1}^{n} b_{i} \hat{f}\left(\beta_{i}\right)=0$ since $\hat{f}\left(\beta_{i}\right)=0$ for $i=1, \ldots, k$. Since the elements of the $B_{\lambda}$ generate $B$ and are contained in the linear space $A^{\perp}$, we have $B \subset A^{\perp}$.

2.3. THEOREM. Let $A$ be a closed subalgebra of $L^{1}(G)$, then

(1) $A_{0}^{\perp}=\left(L^{2}\right.$-closure of $\left.B\right) \cap L^{\infty}(G)$

(2) $A^{0 \perp}=w^{*}$-closure of $B$.

Proof. (i) By (2.2) we have $A^{\perp} \supset B$. Now choose $\phi \in\left(L^{2}\right.$-closure of $\left.B\right) \cap L^{\infty}(G)$. We will show $\phi \in A_{0}^{\perp}$. 
Now, there exists a sequence $\phi_{1}, \phi_{2}, \ldots$ in $B$ with $\left\|\phi-\phi_{n}\right\|_{2} \rightarrow 0$ as $n \rightarrow \infty$. Suppose $P_{\lambda} \in A_{0}$. Applying the Cauchy-Schwarz inequality and using the fact that $\phi_{n}\left(P_{\lambda}\right)=0$ for every $n$, we have

$$
\begin{aligned}
\left|\phi\left(P_{\lambda}\right)\right| & =\left|\int \phi(x) P_{\lambda}(-x) d x\right| \leqq\left|\int\left(\phi(x)-\phi_{n}(x)\right) P_{\lambda}(-x) d x\right|+\left|\phi_{n}\left(P_{\lambda}\right)\right| \\
& \leqq\left\|\phi-\phi_{n}\right\|_{2} \cdot\left\|P_{\lambda}\right\|_{2} \rightarrow 0 \quad \text { as } n \rightarrow \infty .
\end{aligned}
$$

Thus, $\phi\left(P_{\lambda}\right)=0$. Consequently, since linear combinations of the $P_{\lambda}$ are dense in $A_{0}$, we have $\phi \in A_{0}^{\perp}$. This yields

$$
\left(L^{2} \text {-closure of } B\right) \cap L^{\infty}(G) \subset A_{0}^{\perp} .
$$

(ii) Choose $\phi \in A_{0}^{\perp}$. Clearly $\phi \in L^{\infty}(G)$. We will show $\phi \in\left(L^{2}\right.$-closure of $\left.B\right)$. Let $\lambda_{1}, \lambda_{2}, \ldots$ be the indices for which $\hat{\phi}\left(E_{\lambda}\right) \neq\{0\}$ and $\left\{\beta_{1}, \beta_{2}, \ldots\right\}=E_{0} \cap$ support of $\hat{\phi}$. If $E_{\lambda}=\left\{\gamma_{1}, \ldots, \gamma_{n}\right\}$ then $0=\phi\left(P_{\lambda}\right)=\sum_{i=1}^{n} \hat{\phi}\left(\gamma_{i}\right)$. So $\phi_{\lambda}=\sum_{i=1}^{n} \hat{\phi}\left(\gamma_{i}\right) \gamma_{i} \in B_{\lambda} \subset B$. By the Plancherel theorem $\phi$ is the $L^{2}$-limit of the sums $\sum_{i=1}^{k}\left(\phi_{\lambda_{t}}+\beta_{i}\right) \in B$. Hence, $\phi \in\left(L^{2}\right.$-closure of $\left.B\right) \cap L^{\infty}(G)$. We have proven

$$
A_{0}^{\perp} \subset\left(L^{2} \text {-closure of } B\right) \cap L^{\infty}(G) .
$$

Consequently, (i) and (ii) yield (1).

(iii) Using (2.2) we have $A^{0 \perp} \supset B$. But every annihilator in $L^{\infty}(G)$ is $w^{*}$-closed. Thus,

$$
A^{0 \perp} \supset w^{*} \text {-closure of } B \text {. }
$$

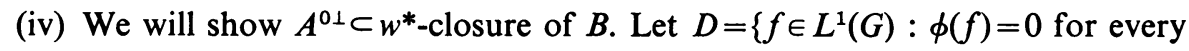
$\phi \in B$ \}. Then $D^{\perp}=w^{*}$-closure of $B$. Hence, it will suffice to show $A^{0} \supset D$, for then

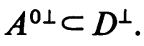

Pick $g \in L^{1}(G)$ with $g \notin A^{0}$. Then either there exists $\gamma_{1} \sim \gamma_{2}$ with $\gamma_{1}, \gamma_{2} \notin E_{0}$ and $\hat{g}\left(\gamma_{1}\right) \neq \hat{g}\left(\gamma_{2}\right)$ or there exists $\beta \in E_{0}$ with $\hat{g}(\beta) \neq 0$. If the former is true, let $\Phi=\gamma_{1}$ $-\gamma_{2} \in B$ then $\Phi(g) \neq 0$ and hence $g \notin D$. Otherwise, let $\Psi=\beta \in B$ and conclude $\Psi(g) \neq 0$. Consequently $A^{0} \supset D$, or

$$
A^{0 \perp} \subset w^{*} \text {-closure of } B \text {. }
$$

Combining (iii) and (iv), we have proven (2).

III. Factorization. If a commutative Banach algebra $B$ has an approximate identity there exists a bounded set $U$ of $B$ such that $x$ belongs to the closure of $x U$ for every $x$ in $B$. It is this property that motivates the following definition.

3.1. Definition. Let $B$ be a commutative Banach algebra. We say that $B$ has a pseudo-identity (p.i.) if and only if $x$ belongs to the closure of $x B$ for every $x$ in $B$.

It is well known that $L^{1}(G)$ has an approximate identity which may be chosen to be a (p.i.) for $L^{p}(G), 1 \leqq p<\infty$. But if $1<p \leqq \infty$, it will follow from the HausdorffYoung theorem that $L^{p}(G)$ has no approximate identity. 
Cohen [1] has shown that any Banach algebra $B$ with approximate identity has factorization, i.e., $B=B \cdot B$. In particular, $L^{1}(G)=L^{1}(G) * L^{1}(G)$. One may ask if this property is transferred to all the closed subalgebras of $L^{1}(G)$. We will use a result of Daniel Rider [6] to prove the existence of a closed subalgebra $A$ without factorization and $A \cap L^{p}(G)$ is $L^{1}$-dense in $A$ for $1<p<2$.

We begin with a definition.

3.2. Definition. Let $1<p \leqq \infty$. A closed subalgebra $A$ of $L^{1}(G)$ is a $p$-algebra if and only if $A \cap L^{p}(G)$ is $L^{1}$-dense in $A$.

Clearly, $A_{0}$ is a $p$-algebra for every $p$ and every algebra $A$.

For what follows, we will adopt the notation: $A^{2}=A * A$ and $A^{j}=A^{j-1} * A$ for $j=3,4, \ldots$, where $A$ is a closed subalgebra of $L^{1}(G)$.

3.3. Lemma. Let $A$ be a p-algebra. Then there exists an integer $k$ such that $A^{m} \subset A_{0}$ for $m \geqq k$.

Proof. Case (i). Assume $p \geqq 2$. Then $A_{0} \subset A=L^{1}$-closure of $\left(A \cap L^{p}(G)\right) \subset L^{1}$ closure of $\left(A \cap L^{2}(G)\right) \subset A_{0}$. Hence, $A=A_{0}$ and so $A^{m} \subset A_{0}$ for every integer $m$.

Case (ii). Assume $1<p<2$. Choose $k$ such that $p^{(k-1)}>q$ where $p^{-1}+q^{-1}=1$. Now suppose $m \geqq k$ and $f=f_{1} * \cdots * f_{m}$ where $f_{j} \in A$ for $j=1,2, \ldots, m$. We will show $f \in A_{0}$. Let $\varepsilon>0$ be given.

Since $A$ is a $p$-algebra, we may choose $h=h_{1} * \cdots * h_{m}$ with $h_{j} \in A \cap L^{p}(G)$ for $j=1,2, \ldots, m$ and $\|h-f\|_{1}<\varepsilon$. If we define $\phi_{j}=\hat{h}_{j}$ for $j=1,2, \ldots, m$ it will follow from the Hausdorff-Young theorem that $\phi_{j} \in L^{q}(\Gamma)$ for each $j$.

Let $K$ be any finite subset of $\Gamma$. Then by the Hölder inequality we have

$$
\begin{aligned}
\sum_{\gamma \in K}|\hat{h}(\gamma)| & =\sum_{\gamma \in K}\left|\phi_{1} \cdots \phi_{m}\right|(\gamma) \leqq\left[\sum_{\gamma \in K}\left|\phi_{1}\right|^{q}(\gamma)\right]^{q-1}\left[\sum_{\gamma \in K}\left|\phi_{2} \cdots \phi_{m}\right|^{p}(\gamma)\right]^{p-1} \\
& \leqq\left\|\phi_{1}\right\|_{q}\left[\sum_{\gamma \in K}\left|\phi_{2} \cdots \phi_{m}\right|^{p}(\gamma)\right]^{p-1}
\end{aligned}
$$

By repeated applications of the Hölder inequality to (1) and the fact that $p^{(m-1)} \geqq p^{(k-1)}>q$ we obtain

$$
\begin{aligned}
\sum_{\gamma \in K}|\hat{h}(\gamma)| & \leqq C\left[\sum_{\gamma \in K}\left|\phi_{m}(\gamma)\right|^{p^{(m-1)}}\right]^{p-(m-1)} \\
& \leqq C\left[\sum_{\gamma \in K}\left|\phi_{m}(\gamma)\right|^{q}\right]^{p-(m-1)} \leqq C\left\|\phi_{m}\right\|_{q}
\end{aligned}
$$

where $C$ is a constant independent of $K$. Since $K$ was arbitrary, it follows that $h \in L^{1}(\Gamma) \subset L^{2}(\Gamma)$. Hence by the Plancherel theorem we have $h \in\left(L^{2}(G) \cap A\right) \subset A_{0}$. Since $\varepsilon$ was arbitrary, we conclude $f \in A_{0}$.

3.4. TheOREM. If $A$ is a p-algebra such that $A \subset L^{1}$-closure of $A^{2}$ then $A=A_{0}$. 
Proof. From (3.3) we may pick $k$ such that $A^{m} \subset A_{0}$ for $m \geqq k$. It follows that $A_{0} \subset A \subset L^{1}$-closure of $A^{2} \subset \ldots \subset L^{1}$-closure of $A^{2^{t}}$ where $t$ is chosen to satisfy $2^{t}>k$. Hence $A=A_{0}$.

The following corollaries are immediate.

3.5. Corollary. If $A$ is a p-algebra with (p.i.) then $A=A_{0}$.

\subsection{Corollary. If $A$ is a p-algebra with factorization then $A=A_{0}$.}

Rider [6] has constructed a closed subalgebra $A$ of $L^{1}(T)$ which is generated by a function $f \in L^{p}(T)(1<p<2)$ such that $A^{0}=A \neq A_{0}$. It follows from (3.5) that $A$ does not have a (p.i.) and from (3.6) that $A$ does not have factorization.

Now let $G$ be a locally compact abelian group and $I$ a closed ideal of $L^{1}(G)$. Define $Z(I)=\{\gamma \in \Gamma: \hat{f}(\gamma)=0$ for every $f \in I\}$. The problem of "spectral synthesis" is concerned with the following question: Do there exist two distinct closed ideals $I_{1}$ and $I_{2}$ of $L^{1}(G)$ such that $Z\left(I_{1}\right)=Z\left(I_{2}\right)$ ? Malliavin [7, p. 172] has shown that this will always be the case if $G$ is not compact. For $G$ compact, $Z(I)=E_{0}$, and it is clear that $Z\left(I_{1}\right)=Z\left(I_{2}\right)$ implies $I_{1}=I_{2}$.

Helson [3] has shown that if $I_{1}$ and $I_{2}$ are two distinct closed ideals of $L^{1}(G)$, $G$ not compact, with $I_{1} \subset I_{2}$ and $Z\left(I_{1}\right)=Z\left(I_{2}\right)$ then there exists a closed ideal $I$ with $Z(I)=Z\left(I_{1}\right)=Z\left(I_{2}\right)$ and $I_{1} \varsubsetneqq I \varsubsetneqq I_{2}$. One is struck with the following question for $G$ compact: Suppose $A$ is a closed subalgebra of $L^{1}(G)$ with $A \neq A_{0}$. Does there exist a closed subalgebra $A_{1}$ with $A \supsetneq A_{1} \supsetneq A_{0}$ ? We will use Rider's example and (3.3) to show that this is not generally the case.

For this purpose, let $A$ be a closed subalgebra of $L^{1}(T)$ with $A \neq A_{0}$ and generator $f \in L^{p}(G), 1<p<2$. Now, functions of the form $h(f)$ where $h$ is a polynomial with $h(0)=0$ are dense in $A$. If $\tilde{f}$ represents the image of $f$ under the quotient map $A \rightarrow A / A_{0}$, we have that the functions $h(\tilde{f})$ are dense in the quotient algebra $A / A_{0}$. Now by (3.3) there exists a positive integer $k$ such that $f^{n} \in A_{0}$ for $n \geqq k$. Since every finite dimensional linear subspace of $A / A_{0}$ is closed, it follows that $A / A_{0}$ is a finite dimensional vector space with generators $\tilde{f}, \tilde{f}^{2}, \ldots, \tilde{f}^{(k-1)}$ and thus has dimension less than $k$.

Suppose we could always find a closed subalgebra $B_{1}$ to satisfy $B \supsetneqq B_{1} \supsetneqq B_{0}$ whenever $B$ was a closed subalgebra with $B \neq B_{0}$. Then by induction we may choose $k$ distinct closed subalgebras of $L^{1}(G)$ say $A_{1}, A_{2}, \ldots, A_{k}$ with $A \supsetneqq A_{1} \supsetneq \cdots \supsetneq A_{k}$ $\supsetneq A_{0}$. But then $A / A_{0} \supsetneq A_{1} / A_{0} \supsetneqq \cdots \supsetneq A_{k} / A_{0} \supsetneqq\{0\}$ implies $A / A_{0}$ has dimension greater than $k$. Contradiction.

\section{BIBLIOGRAPHY}

1. P. J. Cohen, Factorization in group algebras, Duke Math. J. 26 (1959), 199-205.

2. P. Curtis, Jr. and A. Figa-Talamanca, "Factorization theorems for Banach algebras" in Function algebras, Scott, Foresman and Co., Chicago, Ill., 1966.

3. H. Helson, On the ideal structure of group algebras, Ark. Mat. 2 (1952), 83-86. 
4. J.-P. Kahane, "Idempotents and closed subalgebras of $A(Z)$ " in Function algebras, Scott, Foresman and Co., Chicago, Ill., 1966.

5. L. Loomis, Abstract harmonic analysis, Van Nostrand, New York, 1953.

6. D. Rider, Closed subalgebras of $L^{1}(T)$, Duke Math. J. 36 (1969), 105-116.

7. W. Rudin, Fourier analysis on groups, Interscience, New York, 1962.

8. A. Zygmund, Trigonometric Series, 2nd ed., Vols. I, II, Cambridge Univ. Press, New York, 1959.

NORTHWESTERN UNIVERSITY,

EVANSTON, Illinois

Massachusetts InStitute of TechNology,

Cambridge, Massachusetts 